\title{
LA ASISTENCIA PSIQUIÁTRICA EN LA HISTORIA POLÍTICA BRASILEÑA*
}

\author{
Ana Teresa A. Venancio \\ Fundación Oswaldo Cruz, Río de janeiro, Brasil
}

\section{RESUMEN}

Este trabajo analiza las relaciones entre la asistencia psiquiátrica y la historia política brasileña durante diferentes momentos: la segunda mitad del siglo XIX, cuando la creación del primer manicomio sirvió como afirmación del poder imperial; el inicio del siglo XX, cuando la configuración de la psiquiatría ayudaba a la joven República a recorrer el camino hacia una nación civilizada; los años 40, en los que un Estado centralizador dio un fuerte impulso a la asistencia psiquiátrica; y desde finales de los años 70, cuando los avances en la psiquiatría brasileña vinieron de la mano de la redemocratización política.

PALABRAS CLAVE: Asistencia psiquiátrica. Psiquiatría. Historia. Brasil.

\section{PSYCHIATRIC CARE IN BRAZILIAN POLITICAL HISTORY}

\begin{abstract}
This study analyzes the relationship between psychiatric care and Brazilian political history at different points in time: in the second half of the nineteenth century, when the creation of the first asylum served as an affirmation of Imperial power; at the beginning of the twentieth century, when the introduction of psychiatry was part of the new Brazilian Republic's pledge to become a «civilized» nation; in the 1940s, when a centralized Brazilian State boosted the expansion of psychiatric care; and as of the 1970s, in which case the Brazilian psychiatric reform has run parallel to political redemocratization.
\end{abstract}

KEY WORDS: Psychiatric care. Psychiatry. History. Brazil.

* Versión ampliada del trabajo presentado en el Simposio Internacional «Sistemas de atención en psiquiatría y salud mental: condiciones histórico-sociales de producción», realizado en Mar del Plata, Argentina, en abril de 2009, organizado por la Red Iberoamericana de Historia de la Psiquiatría. 


\section{INTRODUCCIÓN}

En este trabajo procuro presentar las relaciones existentes entre el desarrollo de la asistencia psiquiátrica en Brasil y la historia política brasileña a lo largo de cuatro momentos distintos: durante la segunda mitad del siglo XIX, cuando la creación del primer manicomio brasileño contribuyó a la afirmación del poder imperial; las primeras décadas del siglo XX, en las que la configuración de una ciencia psiquiátrica ayudaba a la joven República a establecer el rumbo hacia una nación civilizada; el inicio de la década de 1940, momento en el que la construcción de un «nuevo» modelo de Estado, fuerte y centralizador, dio un gran impulso a la asistencia psiquiátrica; y el final de la década de los 70 e inicio de los 80, cuando la reforma del modelo brasileño de psiquiatría, basada en la idea de ciudadanía del enfermo mental, vino ligada al proceso de redemocratización política, tras veinte años de dictadura militar.

Siguiendo las tendencias historiográficas en materia de psiquiatría surgidas en los últimos años en diferentes contextos nacionales ${ }^{1}$, se intenta demostrar que, históricamente, la asistencia psiquiátrica no trajo consigo solamente lugares para la exclusión y el control social. En primer lugar, tratamos de remarcar que la asistencia psiquiátrica también tomó parte en la construcción de representaciones sociales y en el desarrollo del propio Estado, así como de las políticas públicas en el área de la salud. En segundo lugar, se pretende observar cómo, en ciertos momentos de la historia de la psiquiatría en Brasil, la recepción y la asimilación de ideas y de prácticas psiquiátricas europeas no fue una copia literal, ni tampoco una adaptación distorsionada de los conocimientos y de las acciones generados en Europa. Perfectamente al día respecto de los últimos avances de la psiquiatría europea, los psiquiatras brasileños se preocuparon, en cambio, por cuestiones y temas propios de su sociedad y del momento histórico, creando así respuestas para las cuestiones específicas que se debatían en su país. En este sentido, se procura destacar cómo las políticas públicas en materia de psiquiatría no fueron tanto la expresión de una adhesión a los avances técnico-científicos en este área, cuanto el resultado de su interrelación con la historia política brasileña y con procesos sociales concretos.

1 De acuerdo con Huertas, R. (2001), «Historia de la Psiquiatría, ¿Por qué?, ¿Para qué? Tradiciones Historiográficas y Nuevas Tendencias». Frenia. Revista de Historia de la Psiquiatría, I (1), pp. 9-36. SACRISTÁN, C. (2005), «Historiografía de la Locura y de la Psiquiatría en México. De la hagiografía a la historia posmoderna». Frenia. Revista de Historia de la Psiquiatría, V (1), pp. 9-33. 
No obstante, el análisis de estos argumentos no persigue el objetivo de establecer una periodicidad definitiva de la historia de la psiquiatría en Brasil. Las divisiones temporales de los cuatro momentos históricos aquí presentados son fundamentalmente una hipótesis de trabajo ${ }^{2}$ que, de esta forma, se constituye como parte integrante de los argumentos a demostrar. Sin duda prodrían seleccionarse periodos distintos, dando preferencia a otras líneas de análisis como, por ejemplo, la investigación respecto de la creación y la consolidación de la enseñanza de la psiquiatría en Brasil. Es más, la selección de estos cuatro momentos de la asistencia psiquiátrica en Brasil más bien constituye un punto de partida para ayudar a pensar comparativamente la historia de la asistencia psiquiátrica en contextos nacionales específicos.

\section{El NACIMIENTO DE LA PSIQUIATRÍA EN LA ÉPOCA DEL IMPERIO EN BRASIL}

El primer momento que pretendo analizar es el del surgimiento de la psiquiatría en Brasil, con la creación en 1841 del primer manicomio brasileño, inaugurado solamente once años después, en 1852, bajo el nombre de Hospício de Pedro II, en homenaje al joven emperador de Brasil, Pedro II (18251891), hijo de Pedro I (1798-1834), quien había proclamado la independencia de Brasil del imperio portugués en 1822. El primer manicomio brasileño se inspiró en la experiencia francesa, según la cual la psiquiatría surge como última consecuencia de la creación de los asilos, cuya tutela se disputaban las asociaciones médicas y religiosas a finales del siglo XIX.

Sin embargo, los contextos específicos - francés y brasileño- apuntan hacia diferencias significativas. En el caso francés, la creación del asilo psiquiátrico se vio amparada por el proyecto liberal-burgués instaurado por la Revolución Francesa, sirviéndose de forma más clara de la aportación de los médicos para la creación y la implantación de una política asistencial pública que respondiera a la problemática de la exclusión o inserción social de distintos segmentos de la población. En cuanto al caso brasileño, el primer asilo fue una expresión más del régimen monárquico centralizador, surgido a partir del acuerdo entre las élites dirigentes, habiendo sido instaurado por decreto imperial como uno de los actos conmemorativos de la coronación del nuevo empe-

2 De acuerdo con Stagnaro, J.C. (2006), «Evolución y situación actual de la historiografía de la psiquiatría en la Argentina». Frenia. Revista de Historia de la Psiquiatría, VI, 737, p. 10 . 
rador $^{3}$. La ascensión de la clase médica y de sus propuestas se vería limitada por la afirmación y el mantenimiento de ese poder central monárquico, que tenía en la institución religiosa un importante aliado, gracias a la administración del manicomio por la Santa Casa de la Misericordia de Río de Janeiro. En este contexto, el tema de la asistencia social se materializaba especialmente en la forma de caridad con los desvalidos, en lugar de reafirmar la necesidad de un nuevo contrato social.

Uno de los motivos que se encuentran en el origen de la creación de este primer manicomio, de acuerdo con la historiografía ${ }^{4}$, son las denuncias sobre el estado de abandono en que se encontraban los locos en la ciudad de Río de Janeiro. Algunos médicos admiradores de la higiene y la medicina francesa, miembros en su mayoría de la Sociedad de Medicina y Cirugía de Río de Janeiro, protestaron sobre la situación a través de dicha entidad, así como de los artículos que publicaban en los periódicos especializados en medicina. Los médicos franceses Jean Maurice Fraive y Xavier Sigaud, junto con los brasileños José de la Cruz Jobim y Vicente de Simoni, fueron algunos de los personajes que reivindicaron hacia 1839 la creación de un manicomio que diera acogida a los alienados. Aunque estos ruegos encontraran cabida en el proyecto de consolidación de la monarquía en el Brasil imperial, tal consonancia de ideales no fue suficiente para la conformación de la psiquiatría como campo científico.

De esta forma, la creación del primer asilo en Brasil precedió a la existencia de un cuerpo de conocimiento especializado y con organización institucional. En la época de la inauguración del manicomio, en 1852, las primeras facultades de medicina brasileñas (las de Río de Janeiro y de Bahía) — creadas en 1832 y sucesoras de los antiguos Colegios Médico-Quirúrgicos- poseían precisamente una cátedra de Medicina Legal y, posteriormente, una de Higiene.

3 Machado, R. (1978), A Danação da norma. Medicina social e a constituição da psiquiatria no Brasil, Rio de Janeiro, Graal; TEIXEIRA, M.O. (1997), 'O nascimento da psiquiatria no Brasil' En Cadernos IPUB, $n^{\circ} 8$ - Noção de Pessoa e Institucionalização dos Saberes Psicológicos no Brasil, Rio de Janeiro, Instituto de Psiquiatria, pp. 42-78; (1998). Deus e a Ciência na Terra do Sol: o Hospício de Pedro II e a constituição da medicina mental no Brasil. Tese de doutorado em psiquiatria, Rio de Janeiro, IPUB-UFRJ.

4 Entre distintos autores podemos citar: MoreIRA, J. [1905a] (1955), Notícia sobre a evolução da assistência a alienados no Brasil. Arquivos Brasileiros de Neuriatria e Psiquiatria - ed. Especial - 50 anos, 65-101; MACHAdo (1978); ARRUDA, E. (1995), Resumo Histórico da Psiquiatria Brasileira, Río de Janeiro, Ed. UFRJ; TeIXeIRA (1998); Engel, M.G. (2001), Os Delírios da Razão: médicos, loucos e hospícios (Rio de Janeiro, 1830-1930), Rio de Janeiro, Ed Fiocruz. 
Ésta última era una de las principales áreas de investigación médica. En 1887 surgía la revista semanal Brazil Médico, vinculada a la Facultad de Medicina de Río de Janeiro, y la mayoría de sus textos trataban sobre "higiene pública". En el campo de la divulgación científica también abundaban los estudios sobre medicina legal, aunque fue solo más tarde, en la década de 1920, cuando empezó a crecer el número de trabajos sobre "alienación y enfermedades mentales", como lo demuestran los artículos difundidos en la primera publicación médica periódica en Brasil — Gazeta Médica da Bahia-, lanzada en $1866^{5}$.

Los primeros médicos del manicomio y del Asilo Provisório —activo desde 1841 hasta la inauguración del manicomio - eran los profesores catedráticos de Medicina Legal. En Río de Janeiro, el primer catedrático de Medicina Legal — José Martins da Cruz Jobim (1802-1878) - fue, entre 1841 1852, el encargado del Asilo Provisório. Solo en 1881, en una nueva reforma de la enseñanza médica (decreto $\mathrm{n}^{\circ}$. 3.024), se creó la asignatura «Clínica Psiquiátrica y enfermedades mentales», también bajo la absoluta responsabilidad del catedrático de Medicina Legal de la época, el Dr. Nuno Ferreira de Andrade (1851-1922), que fue director médico del manicomio. En 1882, la nueva ley obligó a organizar oposiciones para la cátedra de Psiquiatría en la Facultad de Medicina de Río de Janeiro, fecha en la que fue seleccionado, y nombrado al año siguiente, el Profesor João Carlos Teixeira Brandão (1854-1921). En 1887, poco antes de la proclamación de la República (1889), Teixeira Brandão sería nombrado también director del manicomio:

«Es posible, por lo tanto, afirmar que la Medicina Legal fue prácticamente la cuna de la psiquiatría brasileña. Esta raíz común que une las dos especialidades no es en modo alguno fortuita. Las relaciones de proximidad y conflicto entre la medicina legal y la psiquiatría, demuestran, de forma ejemplar, la importancia del discurso médico en general, y del psiquiátrico en particular, en la definición de las cuestiones políticas fundamentales para la nueva sociedad que emergía» ${ }^{6}$.

De este modo, cuando tiene lugar el surgimiento de la psiquiatría en Brasil, la ciencia se instaura en el asilo por vía de la Medicina Legal, mientras que la caridad religiosa era lo que regía la asistencia. Ese cuadro cambiaría a partir de la proclamación de la República, ocurrida en 1889, fecha en la que

5 Schwarcz, L.M. (1993). O espetáculo das raças, São Paulo, Companhia das Letras, pp. 199 e 222.

6 Russo, J.A. (1993), Psiquiatria, manicômio e cidadania no Brasil. Em Russo, J. et al. (coord.), Duzentos anos de Psiquiatria, Rio de Janeiro, Relume-Dumará/ Editora da UFRJ, p. 9. 
se volvió a denominar el asilo brasileño como Hospício Nacional de Alienados y dejó de formar parte así de la Santa Casa de la Misericordia (decreto $\mathrm{n}^{\circ}$ 206-A, de 15 de febrero de 1890), pasando a ser responsabilidad exclusiva de las instituciones médicas. Parte de la clase médica estaba entonces involucrada en los nuevos proyectos de organización social, animados por la República, lo que contribuía al propio desarrollo de los profesionales, en cuanto que representantes de los grupos de élite que compondrían dicha organización social. La Psiquiatría era por aquel entonces una asignatura específica de la Facultad de Medicina, responsable de la dirección del único manicomio civil brasileño.

Después de casi diez años, en 1897, Teixeira Brandão deja la dirección del Hospício y también la dirección de la Assistência Médico-Legal de Alienados, creada en 1890 como órgano nacional formulador de una política asistencial para los alienados ${ }^{7}$. En lo que se refiere al Hospicio en el período concreto que va de 1887 a 1903, se carece de investigaciones más profundas. Las fuentes primarias y secundarias reunidas presentan un cuadro de denuncias respecto a las condiciones en las que se encontraba el manicomio y su administración, que se materializó en la alternancia de distintos directores: Márcio Nery, interinamente, de 1898 a 1899; Pedro Dias Carneiro de 1900 hasta su jubilación en 1901; seguido por Antonio Dias Barros, antiguo médico residente y profesor de la Facultad de Medicina, quien dirigió el manicomio hasta 1903.

3. LA PSIQUIATRÍA CIENTÍFICA POR JULIANO MOREIRA Y EL PROYECTO CIVILIZADOR DEL INICIO DEL SIGLO XX.

En 1903 se inicia el segundo período al que he hecho referencia al comienzo de este trabajo. En aquel año, Juliano Moreira (1873-1933) fue nombrado director del Hospício Nacional de Alienados, permaneciendo en el cargo hasta $1930 \mathrm{y}$, durante este tiempo, asumió también la dirección de la Assistência a Alienados. Su nombramiento y las reformas que allí emprendió estaban en consonancia con el proceso de urbanización de la ciudad de Río de

7 En 1927, la Asistencia Médico-Legal de Alienados era designada nuevamente como Servicio de Asistencia a Psicópatas (SAP) por el decreto $\mathrm{n}^{\circ} .17 .805$ del 23 de mayo, como sección del Ministerio de Justicia y Asuntos Internos, y en 1930 pasa a formar parte del Ministerio de Educación y Salud Pública, creado por el Governo Provisório. El 2 de abril de 1941, el decreto ${ }^{\circ}$. 1.371 sustituye el SAP por el Servicio Nacional de Enfermedades Mentales (SNDM). ENGEL (2001), p. 258. 
Janeiro (entonces capital federal de Brasil) ${ }^{8}$. Muchas fueron las medidas de saneamiento propuestas e implantadas por Oswaldo $\mathrm{Cruz}^{9}$, director general de salud pública del país durante la gestión del alcalde Francisco Pereira Passos (1902-1906), que consideraban a la ciudad un laboratorio y un modelo de urbanidad a la vez.

Dentro de este contexto de modernización - aunque la idea de país se redujera a la capital federal-, Juliano Moreira tomó parte en los esfuerzos de mejora emprendidos en el área de la salud, acometiendo un conjunto de acciones dirigidas a la ampliación de la asistencia psiquiátrica en Brasil, por la vía de la legislación y del establecimiento de instituciones consideradas como apropiadas. En el primer aspecto, incentivó y defendió la promulgación de la primera Ley federal de asistencia a los alienados (1903), como se puede comprobar en su respuesta al médico Nina Rodrigues (1862-1906), que criticaba la citada ley en el artículo «La asistencia médico-legal a alienados en los estados brasileños», publicado en la Revista Brasil Médico en $1906^{10}$. En cuanto a las instituciones responsables del tratamiento psiquiátrico, la cuestión se debatió en varios artículos entre 1905 y $1910^{11}$. Este último presentaba tanto una revisión histórica acerca del modo como en que los locos y alienados eran tratados en Brasil desde el período colonial (incluyendo un panorama sobre cómo se encontraban en aquel momento las instituciones existentes), como la publicación de una serie de propuestas institucionales y terapéuticas que, a ejemplo de los países europeos, podrían ser de gran valor para el progreso de la asistencia, caso de ser implantadas en Brasil.

8 Sobre el proyeto de urbanización de Río de Janeiro véase: BENCHIMOL, J.L. (2003) Reforma urbana e revolta da vacina na cidade do Rio de Janeiro. In: Jorge Ferreira; Lucilia de Almeida Neve. (Org.). Brasil republicano. Economia e sociedade, poder e política, cultura e representações. Rio de Janeiro, Editora Civilização Brasileira vol 1, pp. 231-286.

9 AzEvedo, N. (1995), Oswaldo Cruz: a construção de um mito da ciência brasileira. Rio de Janeiro, Editora Fiocruz.

10 Moreira, J. (1907a), A lei federal de assistência a alienados e a crítica do professor Nina Rodrigues. Brazil-Medico, ano XXI, n.23, 221-225, 15 de jun.. (1907b) A lei federal de assistência a alienados e a crítica do professor Nina Rodrigues (conclusão). BrazilMedico, ano XXI, n. 24, 231-233, 22 de jun.

11 Moreira, J. (1905b), Assistência aos epilépticos: colônias para eles. Archivos Brasileiros de Psychiatria, Neurologia e Sciencias Affins, Anno I, n.2; MoreIRA [1905a] (1955); . (1908), Ligeira vista sobre a evolução da assistência a alienados na Alemanha. Comunicado a Academia Nacional de Medicina do Rio de Janeiro em nov. 1907, Archivos Brasileiros de Psychiatria, Neurologia e Medicina Legal, n.1 e n. 2. (1910), Quaes os melhores meios de assistência aos alienados. Arquivos Brasileiros de Psiquiatria, Neurologia e Medicina Legal, ano VI, $\mathrm{n}^{\mathrm{o}} 3-4$ 
Ya en los primeros años de su gestión como director, Juliano Moreira dotó de nuevo equipamiento al Hospício Pedro II, institución de finales del siglo XIX, en esos momentos ya vista como obsoleta y estancada. Fundó los laboratorios de bacteriología y virología priorizando - en lugar de los chalecos, las camisas de fuerza y las rejas - el uso de oficinas de trabajo, clinoterapia y open door ${ }^{12}$. Persiguió también la reforma de las colonias agrícolas de la Ilha do Governador (Isla del Gobernador, barrio de Río de Janeiro), creadas en 1890, así como la construcción de nuevas colonias para enfermos crónicos y para las víctimas de enfermedades específicas: alcoholismo, sífilis, lepra, etc. Al parecer, Juliano quería para Brasil, a ejemplo de los «países más civilizados», una «asistencia diferenciada de acuerdo con las distintas formas de enfermedades cerebrales, creando por tanto hospitales-colonias especiales para epilépticos, para alcohólicos y para minusválidos, imbéciles e idiotas» ${ }^{13}$.

Quedaba mucho por construir y, en este sentido, Juliano Moreira escribió insistentemente a favor de las colonias ${ }^{14}$. Su principal argumento era que se hacía necesario ofrecer asistencia a los desamparados, especialmente a los más pobres, por los mayores riesgos a que se veían expuestos a consecuencia de la degeneración causada por tales enfermedades. Defendía que todos estos enfermos podrían beneficiarse en alto grado de la vida al aire libre, combinada con el trabajo en el área de la asistencia heterofamiliar. La inspiración, como ocurría con los países europeos, era la pequeña aldea de Gheel; sin embargo, Juliano Moreira consideraba que era imposible reproducir esa experiencia que, según él, se había iniciado en el siglo XVII con los aldeanos de Gheel hospedando a locos que creían en una curación milagrosa en la Iglesia local de Santa Dymphne. De esta forma, apostaba por el llamado «sistema alemán», en el que la asistencia de los colonos de Gheel era sustituida por la asistencia familiar, llevada a cabo fuera de la institución por empleados y familias ${ }^{15}$. Este proyecto se plasmaría en Río de Janeiro con la inauguración, en 1924, de la Colônia de Psicopatas-Hombres, ubicada en la zona rural de Jacarepaguá,

12 La defensa, por Juliano Moreira, del open-door, de la clinoterapia y del régimen heterofamiliar, está presente en MoREIRA, J. (1901) Klinoterapia. Diffusão do seu uso, sua technica e resultados no tratamento das psychoses. (extraído do Brazil-Medico). Rio de Janeiro: Typographia Besnard Frères,. (1906) Ligeiras Notas a Propósito da Assistencia Familiar, Archivos Brasileiros de Psychiatria, Neurologia e Sciencias Affins, Anno II, $\mathrm{n}^{\mathrm{o}}$ 1, jan. e MOREIRA, J. [1905] (1955); (1910).

13 MOREIRA 1910, p. 387.

14 En MOREIRA (1910) se observa el uso de varias categorías para hablar sobre el espacio colonial que se quería implantar: colonia agrícola, asilo-colonia, hospital-colonia.

15 MOREIRA 1910, p.386. 
adonde fueron llevados los pacientes de las antiguas colonias de la Ilha do Governador ${ }^{16}$.

Esas inversiones en el área asistencial venían ligadas al desarrollo de una ciencia psiquiátrica brasileña, produciéndose, también por esa vía, la inclusión de Brasil en el conjunto de las naciones civilizadas. Con objeto de integrar la psiquiatría en los movimientos a favor del «progreso» de las ciencias en el país, Juliano Moreira fomentó la difusión de publicaciones periódicas científicas, como lo hizo él mismo, con la creación en 1905, juntamente con el médico forense Afranio Peixoto, de los Archivos Brasileiros de Psychiatria, Neurología e Ciências Affins ${ }^{17}$, órgano de divulgación de la primera sociedad científica psiquiátrica brasileña: Sociedade Brasileira de Psychiatria, Neurología e Ciências Afins. También defendió e implantó el uso de lenguajes y sistemas especializados, como la primera clasificación psiquiátrica brasileña en 1910, participando en congresos médicos internacionales (Lisboa, 1906; Ámsterdam y Milán, 1907; Londres y Bruselas, 1913).

Respecto a las teorías psiquiátricas, Juliano Moreira se vio claramente influenciado por el alemán Émil Kraepelin y sus teorías de corte organicista. Al igual que Kraepelin, Juliano Moreira entendía la enfermedad mental como un estado de naturaleza distinta al de los estados considerados normales. La concebía como un fenómeno limitado al plano de la «excepción biológica», y adoptaba los principios clasificatorios propuestos por el psiquiatra alemán: los síntomas observados en los términos de su etiología y la evolución de la enfermedad. En opinión de Juliano Moreira, la enfermedad mental «como desviación de la normalidad, es una excepción biológica» ${ }^{18} \mathrm{y}$, en ese sentido, solo puede observarse a partir de la consideración preponderante de la esfera orgánica del individuo. Como en Kraepelin, se trataba de una síntesis entre la

16 Sobre la historia de esa instituición, en 1935 nuevamente denominada Colônia Juliano Moreira véase VenAncio, A.T.A. ; CASSILIA, J.A.P. (2010), Política assistencial psiquiátrica e o caso da Colônia Juliano Moreira: exclusão e vida social (1940-1954). In: WADI, Y.M; SANTOS, N.M.W. (orgs.) História e Loucura: saberes, práticas e narrativas. Uberlandia, EDUFU, pp. 51-83.

17 Muchos de los artículos escritos por él a favor de las transformaciones en la asistencia psiquiátrica, aquí ya citados, fueron publicados en este periódico. Inicialmente nombrado Arquivos Brasileiros de Psiquiatria, Neurologia e Ciências Afins (abril 1905-1907), después bautizado nuevamente como Arquivos Brasileiros de Psiquiatria, Neurologia e Medicina Legal, (1908-1918) y, por último, como Arquivos Brasileiros de Neuriatria e Psiquiatria (1919-1957?)

18 MoreIRA, J. (1919), Classificações em Medicina Mental. Archivos Brasileiros de Neuriatria e Psychiatria, Anno $1,1^{\circ}$ trimestre, p. 93. 
etiología moral y física solo en la medida en que se consideraba que la etiología física podría abarcar y designar una probable etiología moral. La causalidad, por consiguiente, se relaciona con la acción de ciertas toxinas sobre el córtex cerebral, asociándose a perturbaciones generales del organismo que se manifiestan en síntomas.

En ese modelo eminentemente médico, los síntomas de las enfermedades mentales se relacionaban con afecciones de la voluntad y de la conciencia, consideradas desde el punto de vista organicista, como lo había hecho Kraepelin:

«(...) los estados depresivos no son más que la conciencia del estado del cuerpo, de la hipotonía de los músculos lisos y estriados, el resultado de la desnutrición muscular y cerebral; se espera que la mejora del estado general, la reparación de las perturbaciones nutritivas del organismo y del cerebro, cambien el estado kinestésico que provoca en la conciencia una conmoción dolorosa. Y la hiperestesia psíquica del melancólico, con sus dolores morales angustiosos, con su estado abúlico acentuado, necesita con urgencia algo que le suprima la actividad de los músculos relacionales de la vida, por ello el paciente necesita que se le ahorre todo acto voluntario, cualquier determinación propia.» ${ }^{19}$

Como en la teoría kraepeliniana, la visión de Juliano Moreira sobre la voluntad y la conciencia no estaba en relación con ninguna consideración de orden moral que pusiese en cuestión el libre albedrío individual o la existencia de una motivación idiosincrática. Se trataba en exclusiva de la conciencia y de la voluntad puestas en juego en las reacciones motoras, del sustrato físico propiciador o imposibilitador de la acción psicomotora individual; esa conciencia y voluntad de carácter físico eran determinantes para la expresión de las capacidades de afecto, de juicio y, por consiguiente, del total individual que representa la personalidad ${ }^{20}$. Desde este punto de vista, J. Moreira se centró en el análisis de diagnósticos cuya descripción tuviese en cuenta aquellos fenómenos de intoxicación que afectasen a la dimensión orgánica individual - las neuronas, el sistema nervioso- y que generasen comportamientos

19 Moreira, J. (1901), Klinoterapia. Diffusão do seu uso, sua technica e resultados no tratamento das psychoses., Rio de Janeiro,Typographia Besnard Frères, p.43.

20 Podemos entender, en este sentido fisicalizador, la referencia que hacen Juliano Moreira y Afrânio Peixoto sobre las llamadas intoxicaciones crónicas, de las que el Prof. Kraepelin «solamente se ocupó de las llamadas voluntarias: alcoholismo, morfinismo y cocainismo» Moreira, J. e PeiXoto, A. (1905), Classificação de molestias mentaes do professor Emil Kraepelin. Archivos Brasileiros de Psychiatria, Neurologia e Sciencias Affins, Anno I, n. 2, pp. 204-216. 
«anormales» a los sujetos: la lepra, la sífilis ${ }^{21}$ y el alcoholismo. De esta forma, buscaría, por ejemplo, hacer patente la confluencia de psicopatías entre los leprosos, no con vistas a establecer una relación de causa y efecto entre la segunda y la primera enfermedad, sino para afirmar que «no se puede negar que las toxinas leprosas actuando sobre las neuronas superiores de un individuo predispuesto, pueden producir psicosis infecciosas ${ }^{22}$.

Las teorías kraepelinianas servirían a Juliano Moreira para responder a cuestiones específicas, surgidas en aquel momento, acerca del horizonte y las futuras vías de desarrollo de la sociedad durante la Republica brasileña. En este sentido, paradójicamente, las teorías psiquiátricas alemanas fueron «traducidas» por Moreira en un discurso anti-determinista, contrario a la creencia en la inexorabilidad de los males generados por el clima tropical y por la constitución racial de los brasileños:

«El clima no influye en nada sobre los síntomas de diversas psicosis. Es en el grado de formación del individuo donde reside la causa de las diferencias que puedan presentarse. El descendiente puro de dos caucasianos, igualmente puros, criado en el interior en medio a personas ignorantes, presenta los mismos delirios rudimentarios que los individuos de color desprovistos de formación» ${ }^{23}$.

Afirmaba que no se debería atribuir las enfermedades mentales que afectaban a la población brasileña a esos factores ambientales o raciales, sobre los cuales nada se podría hacer. Para él, el motivo de las enfermedades mentales en Brasil, igual que en todos los países del Viejo Mundo, se encontraba en el plan biológico estrictamente individual, y no en el plan colectivo o social. En la discusión propiciada por las teorías médico-legales racistas de finales del siglo XIX - expresadas en el pensamiento de Nina Rodrigues (1862-1906), por ejemplo - Juliano Moreira proponía el igualitarismo de las razas, afirmando ser posible la inclusión del mestizo pueblo brasileño en un proyecto civilizador universal, por medio de acciones públicas en la área de la salud y de la educación.

21 MoreIRA, J. (1918), A Psychiatria e a Guerra. Conferência realizada no Club Militar em 4-2-1918 sob os auspicios da Sociedade Medico-Cirurgica Militar. Capital Federal, apud Portocarrero, V.M. (2002), Juliano Moreira e a descontinuidade histórica da psiquiatria, Rio de Janeiro, Editora Fiocruz, p.87.

22 Moreira, J. (1906), Psychoses em leprosos. Archivos Brasileiros de Psychiatria, Neurologia e Sciencias Affins, Anno II, n ${ }^{\circ}$, p. 54.

23 Moreira, J. e Peixoto, A. (1906), Les maladies mentales dans les climats tropicaux. Archivos Brasileiros de Psychiatria. Neurologia e Sciencias Affins, Anno II, (1), p. 238. 


\section{LA ASISTENCIA PSIQUIÁTRICA EN EL CONTEXTO DE LAS POLÍTICAS DE SALUD EN BRASIL (1937-1954)}

El tercer momento que quiero destacar se inicia en 1937, cuando, por motivo de un golpe de estado, el Gobierno Federal bajo la presidencia de Getúlio Vargas (1882-1954) instauraba un régimen de excepción política denominado Estado Novo. Getúlio Vargas había alcanzado la Presidencia de la República de Brasil en 1930, por medio de un golpe sostenido por un levantamiento militar que, bajo su liderazgo civil, tenía como objetivo impedir la investidura del nuevo presidente - Júlio Prestes - elegido en marzo del mismo año. Las causas de este movimiento armado se venían gestando ya desde décadas anteriores, como cabe constatar: (i) por una parte, la alternancia política en los presidentes de la República, representantes bien del Estado de São Paulo, bien del de Minas Gerais $^{24}$, lo que favoreció desde la década de 1910 a las oligarquías de ambos estados, en detrimento de otras oligarquías locales sin acceso a los centros de decisión nacionales, e hizo emerger un descontento creciente en éstas; (ii) y por otra parte, en el surgimiento de nuevas fuerzas sociales en Brasil desde los años 20, en especial de una clase media, como consecuencia de los procesos de industrialización y urbanización, la cual comenzaba a exigir una participación y una representación políticas que, hasta el momento, no había experimentado.

Getúlio ascendió al poder apoyado por las oligarquías de distintos estados y por la clase media urbana, teniendo a la vez como desafíos políticos el ser representante de los anhelos de las fuerzas sociales que lo apoyaron (clases medias urbanas y oligarquías locales), y resolver la tensión entre los proyectos políticos de centralización y los de reparto del poder entre las instituciones nivel local. Ambos proyectos ocasionaban disputas entre las oligarquías ${ }^{25}$. En este contexto el gobierno de Getúlio Vargas (1930-45) buscó un nuevo mode-

24 Esa alternancia de presidentes procedentes de los estados de São Paulo y Minas Gerais se dio a conocer como política del «café con leche», considerándose que los dos estados eran, respectivamente, los mayores productores de café y de leche. En el lenguaje popular brasileño la expresión "ser café con leche» significa que el sujeto en cuestión no se encuentra en situación de competencia igualitaria con los demás, siendo considerado como miembro elegido o integrante de un determinado ámbito sin haber pasado por los trámites de evaluación o competencia a los que se someten los demáss indivíduos.

25 Fonseca, C.; Hochman, G.; Trindade, N.L. (2005), A Saúde na Construção do Estado Nacional no Brasil: Reforma Sanitária em Perspectiva Histórica. In: LIMA, N.T.; GERSCHMAN, S.; EDLER, F.C.; SUÁREZ, J.M. (coords.), Saúde e Democracia: História e Perspectivas do SUS, Rio de Janeiro, Editora Fiocruz, pp. 27-58, p. 38 e ss. 
lo para las instituciones políticas y para la estructura del sistema de salud pública, donde se encontraba la asistencia psiquiátrica.

A partir de 1937, con el Estado Novo, el Gobierno Federal expresa su clara opción por una política centralizadora, liderada solamente por las instancias federales, y que se inclinaba tanto hacia la modernización del Estado, como hacia la construcción de un proyecto nacional. Dentro de ese proceso de mayor centralización política y administrativa, todos los órganos del Ministerio de Educación y Salud Pública, con competencias en el área de la salud, pasaron a integrar el Departamento Nacional de Salud (DNS) ${ }^{26}$. Se incluían aquí aquellos que hasta ese momento estaban bajo la responsabilidad de la División de Asistencia a Psicópatas, encargada de las siguientes tareas:

«de los servicios concernientes a la asistencia a psicópatas y a la prevención mental, de carácter nacional, así como de aquellos de carácter local que sean ejecutados por el Gobierno Federal; de su responsabilidad será promover la cooperación del Gobierno Federal en los servicios locales, por medio del auxilio y de la subvención federales, fiscalizando el empleo de los recursos concedidos. $\gg^{27}$

Durante ese período se verificó en el campo de la salud un proceso tanto de centralización política como de descentralización en lo referente a la implantación efectiva de las decisiones del Estado, buscando una interacción entre los ámbitos federal, regional y municipal de gobierno. Las decisiones del Gobierno Federal seguían las directrices para las políticas de salud pública que se discutían internacionalmente. Entre 1930 y 1945, la Organización Panamericana de Salud había promovido varias reuniones recomendando el modelo de «centralización normativa y descentralización directiva». Con ese objeto fueron creados los llamados «distritos sanitarios»— áreas que comprendían grupos de municipios - por medio de los cuales se realizaría el control de las acciones de sa$\operatorname{lud}^{28}$. La intención era instituir la fiscalización de los municipios bajo la responsabilidad de los estados, garantizando jerárquicamente el control federal sobre todas las instancias, no obstante, sin dejar de dialogar con los poderes locales, que mantenían de esa manera alguna relevancia en el juego de poder.

Asimismo, desde 1941 se institucionalizó la reforma del Departamento Nacional de Salud, que distribuyó sus áreas de acción según enfermedades.

26 El Departamento Nacional de Salud estaba, por lo tanto, compuesto por cuatro secciones: División de Salud Pública, División de Asistencia Hospitalaria, División de Amparo a la Maternidad y a la Infancia y la División de Asistencia a Psicópatas.

27 BRASIL. Artigo $17^{\circ}$ da Lei n ${ }^{\circ} 378$ de 13 de janeiro de 1937.

28 FONSECA, et. al.(2005), p.45 
Tales áreas de acción pasaron entonces a ser responsabilidad de distintos órganos: Servicio Nacional de Lepra, Servicio Nacional de Malaria, Servicio Nacional de Peste, Servicio Nacional de Tuberculosis y Servicio Nacional de Fiebre Amarilla ${ }^{29}$. En ese contexto fue creado también el Servicio Nacional de Enfermedades Mentales (SNDM), que reunía la División de Asistencia a Psicópatas (DAP) de alcance nacional y el Servicio de Asistencia a Psicópatas, orientado al Distrito Federal. El Departamento Nacional de Salud ampliaba su acción en el área psiquiátrica, hasta entonces exclusivamente concentrada en la capital federal. La tarea de formulación de una política asistencial psiquiátrica de ámbito nacional salía así fortalecida.

En ese contexto, de 1937 a 1941, el gobierno federal realizó una investigación que tenía como objetivo evaluar las condiciones de la asistencia psiquiátrica, obteniendo como resultado una clasificación de los servicios prestados por los diferentes regiones brasileñas: en los estados de Sergipe, Goiás y el territorio de Acre la asistencia era considerada inexistente; en Mato Grosso, Espírito Santo y Piauí la asistencia fue evaluada como deficiente, sin tratamiento diferenciado y especializado; en Amazonas, Maranhão, Ceará, Río Grande do Norte, Alagoas y Santa Catarina, se constató que había alguna oferta de orientación especial, pero con asistencia muy deficiente; en Paraíba, Pará, Bahía y Río de Janeiro la asistencia se entendió como especializada, pero reducida. Solo en cinco estado brasileños - Paraná, Rio Grande do Sul, Pernambuco, São Paulo y Minas Gerais - los métodos psiquiátricos fueron considerados más modernos y dirigidos a la prevención ${ }^{30}$.

En base a esa investigación, desde 1941 el Servicio Nacional de Enfermedades Mentales pasaría a gestionar la extensión de la asistencia psiquiátrica a todo el territorio nacional, creando el Plan Hospitalario Psiquiátrico, cuyo objetivo era la planificación e instalación de 4.000 plazas psiquiátricas en diferentes estados, con la ayuda financiera del Gobierno Federal. Entre las propuestas del plan se observa una insistencia en el modelo institucional de 'hospital-colonia'; bajo la directriz de que una «futura acción - como la que se proyecta - no se vea en la obligación de abandonar lo hasta ahora realizado» ${ }^{31}$. De entre los veinte estados brasileños existentes en la época (incluyén-

29 BRASIL. Decreto-lei n ${ }^{\circ} 3.171$ de 2 de abril de 1941.

30 Pereira, M. de F. (1992), A psiquiatria brasileira nos anos de 1930 a 1950. (dissertação de mestrado, não defendida), Rio de Janeiro, IMS/UERJ, p.45-6

31 BRASIL/Ministério da Educação e Saúde /Departamento Nacional de Saúde (DNS). Plano Psiquiátrico para a União Sugestões para a Ação Supletiva da União. In: Arquivo Gustavo Capanema, série Ministério da Educação e Saúde — Saúde e Serviço Social, GCh 
dose la capital federal), catorce habían sido señalados para la construcción o la ampliación de un hospital-colonia o colonia. Para los demás estados (Amazonas, Pará, Río Grande do Norte, Paraíba, Bahía y Paraná) se preveía la construcción de hospitales o de pabellones en sus interiores.

Ese modelo institucional de colonias para pacientes psiquiátricos no era una novedad en Brasil, igual que sucedía en otros países iberoamericanos y europeos. Como hemos visto anteriormente, la noción de colonia y sus derivados semánticos (asilo-colonia, colonia agrícola, hospital-colonia), destinada incluso a diferentes tipos psicopatológicos (alcohólicos, epilépticos), era defendida desde el inicio del siglo XX por Juliano Moreira. En este nuevo contexto entre los años 1940 a 1950, el modelo de la colonia aparecía asociado al servicio del hospital moderno, sirviendo como «modelo», tanto por considerar lo que había de más adecuado para el tratamiento de la enfermedad mental, como en lo relativo al incentivo sistemático que se ofrecía por tomarlo como patrón. El prototipo del hospital-colonia se concebía entonces como un complejo hospitalario que ocupaba un amplia área física, apartado de los centros urbanos, integrado por grandes pabellones y núcleos para el ingreso de cientos de pacientes, quiénes, a su vez, tendrían como recursos terapéuticos la antigua praxisterapia, pero también los modernos tratamientos de convulsoterapia. Con ese modelo, el Estado brasileño creía promover la centralización y la modernización de la asistencia psiquiátrica a nivel nacional.

El principal personaje de este proceso fue Adauto Botelho (1895-1963), médico licenciado por la Facultad de Medicina de Río de Janeiro en 1917 y primer director del Servicio Nacional de Enfermedades Mentales, que se mantuvo en el cargo hasta 1954. Desde el punto de vista de la clínica, se interesaba particularmente por el tema del alcoholismo. En 1921, juntamente con los psiquiatras Pedro Pernambuco, Antonio Austregésilo y Ulisses Vianna, había fundado el Sanatorio Botafogo: una clínica privada con menos de 20 plazas psiquiátricas, orientada al tratamiento de las toxicomanías, en un momento en que éstas se presentaban como categoría diagnóstica en círculos psiquiátricos de la ciudad de Río de Janeiro. Además de ello, su producción académica, aunque reducida cuantitativamente frente a otros psiquiatras de renombre, se centró en el tema del alcoholismo, y en otros como los de la parálisis general, la psicosis maníaco-depresiva, el síndrome de Ganser, además de otros dos trabajos sobre política asistencial, todos publicados en periódicos especializados de Río de Janeiro, capital de la República. En los Ar34.08.03 / II-14. Rio de Janeiro, Centro de Pesquisa e Documentação de História Contem-
porânea do Brasil /Fundação Getúlio Vargas (CPDOC/FGV). s/d 
chivos Brasileiros de Psychiatria, Neurologia e Sciencias Affins publicó ocho artículos, mientras que en el Archivos Brasileiros de Higiene Mental fueron tres artículos. En el Jornal Brasileiro de Psiquiatría, órgano oficial de divulgación del Instituto de Psiquiatría de la Universidad de Brasil — de la que había sido director (1956-1958) — publicó solamente el artículo «Influencia del psicoanálisis sobre la psiquiatría» (1956).

Como responsable del Servicio, Adauto Botelho fomentó la implantación de hospitales-colonia en varias capitales de los estados y creó el Centro Psiquiátrico Nacional (hospital psiquiátrico, colonias, hospital para niños y para neurosífilis) posteriormente llamado Centro Psiquiátrico Pedro II. En 1944, iniciaba de modo incipiente la difusión de los Ambulatórios de Saúde Mental, desde el Consultório de Psico-Higiene en Río de Janeiro. Al final de su gestión de 13 años, Adauto Botelho había realizado una ampliación de 16000 plazas psiquiátricas en todo el país ${ }^{32} \mathrm{y}$, no sin razón, muchas de las nuevas colonias y hospitales fueron inaugurados con su nombre. Basados en estos modelos, por ejemplo, fueron creados el Hospital Psiquiátrico Adauto Botelho en Cuiabá, y el Hospital Colonia Adauto Botelho en Cariacica (Espírito Santo), cuyas obras fueron iniciadas en 1949; y el Hospital Colonia Adauto Botelho $^{33}$ en Pinhais (Paraná), que empezó a funcionar en 1954. También la Colônia Santana, establecida a finales de 1941 en el municipio de San José, en el Salto do Imaruí (Santa Catarina), formó parte de esa directriz asistencial psiquiátrica establecida por el Dr. Adauto Botelho. Un número significativo de estas plazas psiquiátricas se implantó en base al Decreto Ley 8.550, de 1946, que autorizaba el SNDM a realizar convenios con los estados de la federación. El Gobierno Federal se encargaría de la construcción y dotación del nuevo centro psiquiátrico, mientras que las instituciones de cada estado estarían encargadas de la donación del terreno, de los gastos de mantenimiento de las instalaciones y de la retribución de los profesionales que allí actuarían.

A este respecto, lo que se observa es que la política psiquiátrica durante este período, que fue responsable del ingreso de un número significativo de personas, no solo acrecentó el proceso de exclusión social de muchos individuos y de grupos amplios de la población, sino que también contribuyó al establecimiento del propio Estado brasileño, por medio de la planificación y

32 De acuerdo con Paulin, L.F. e Turato, E.R. (2004), Antecedentes da reforma psiquiátrica no Brasil: as contradições dos anos 1970. História, Ciências, Saúde- Manguinhos, vol. 11(2), 241-58, p.244.

33 SigOLO, R. P. (2001), Vislumbrando o diferente: teorias psiquiátricas na formação da Colônia Santana. Psychiatry on line Brazil, vol. 6 (4). http://www.polbr.med.br/ano01/wal0401.php 
la ejecución político-administrativa de los órganos encargados, favoreciendo el liderazgo del Gobierno Central frente al de los estados de la Federación.

\section{LA REFORMA PSIQUIÁTRICA BRASILEÑA DESDE FINALES DE LA DÉCADA DE 1970}

En la época reciente, los nuevos rumbos de la asistencia psiquiátrica en Brasil están en relación con otro momento específico de la historia política brasileña, a finales de la década de 1970 e inicio de los años 80. Se trata de una época marcada por un proceso de distensión de la represión política, instaurada con el régimen militar de 1964, y cuya intensificación se materializó en la implantación del Acto Institucional no 5 en 1968. A finales de los años 1970, el proceso paulatino de redemocratización política supuso la vuelta de las agrupaciones políticas a la legalidad, la creación de nuevos partidos, como el Partido de los Trabajadores (1978), y el surgimiento de movimientos sociales en conexión con diferentes grupos de minorías y las condiciones de vida generales de la población, como las asociaciones de barrios y de favelas.

Por una parte, el modelo de desarrollo sostenido por los gobiernos militares estaba roto y, por otra, la sociedad civil volvía a organizarse reivindicando la normalidad de los derechos políticos y la amnistía para los exiliados. En el área de la salud se observa la denuncia y la crítica de la política vigente, marcada por el abandono de las instituciones asistenciales públicas y por la excesiva privatización de los servicios, que el Estado financiaba pero no controlaba. En cuanto a la asistencia psiquiátrica, esta práctica fue llamada más tarde «industria de la locura» ${ }^{34}$. Por lo menos desde la década de 1960, el Gobierno Federal había dejado de invertir en la compra de equipos y en la renovación de las instituciones públicas del área psiquiátrica, manteniendo el modelo centrado en la hospitalización por medio del pago de plazas en instituciones privadas. Sin embargo, el pago de plazas privadas no vino acompañado de una fiscalización de los servicios de salud financiados. De esta forma, junto al abandono de las grandes instituciones psiquiátricas públicas, se pudo observar una falta de criterios y de fiscalización técnico-terapéutica en los servicios privados, convirtiéndose así en nuevos lugares de depósito de personas y de creación de conductas crónicas en los enfermos mentales.

34 De acuerdo con RuSSO, J.A.; VENANCIO, A.T.A. (2008), Loucura e Responsabilidade. In: Souza Lima, A.C.; SchritzmeYer, A.L.P.; CARrara, S. (coords.). Antropologia e Direito: bases para um diálogo interdisciplinar (NO PRELO). Blumenau, Nova Letra. 
En ese contexto político y en contra la «industria de la locura», tuvo origen, al final de la década de 70, un proceso que fue conocido como la reforma psiquiátrica y que, inicialmente, estuvo vinculado al escenario de la reforma sanitaria propuesta por los médicos por entonces comprometidos en la crítica al régimen militar. Para la reforma sanitaria, el asunto de la ciudadanía se confundía con el de la extensión universal del acceso a los bienes sociales, o sea, la extensión igualitaria de servicios de salud de buena calidad a toda la población.

La reforma psiquiátrica brasileña, a su vez, tuvo como punto de origen, en 1978, la «crisis de la DINSAM» - División Nacional de Salud Mental, órgano federal encargado de la política de salud mental del país, creado en 1970 en sustitución del Servicio Nacional de Enfermedades Mentales. Los profesionales del área manifestaron las pésimas condiciones de los tres hospitales psiquiátricos del Ministerio de la Salud localizados en Río de Janeiro y divulgaron una denuncia corporativa de explotación del trabajo de los denominados «becarios»: profesionales y estudiantes que trabajaban en tales instituciones cobrando «becas de salud mental», es decir, un tipo de remuneración que no veía reconocidos los derechos laborales garantizados por el estado brasileño desde los años 30 . Como respuesta, el gobierno prescindió de los becarios, a continuación extinguió los programas de prácticas y trabajo voluntario existentes en las tres instituciones, y contrató médicos que habían superado las oposiciones realizadas por el Ministerio de Seguridad y Asistencia Social, para sustituirlos. En el mismo año se realizó en la región sur del país, en la ciudad de Camboriú, el V Congreso Brasileño de Psiquiatría, que contó con la presencia de un grupo de profesionales que habían sido cesados por la DINSAM, dando a ese congreso técnico-científico una connotación eminentemente política en cuanto a las direcciones a seguir por la psiquiatría brasileña. Los profesionales se reunieron en una asamblea general y lanzaron el «Manifiesto de Camboriú», fijando para enero de 1979 un Encuentro de Profesionales de Salud Mental ${ }^{35}$.

Esta movilización política en el área psiquiátrica creó como lema la expresión «por una sociedad sin manicomios». Se inspiraba principalmente en la experiencia italiana liderada por Franco Basaglia, que denunciaba las pésimas condiciones de trabajo de los profesionales de la salud mental y las situaciones inhumanas en las que vivían los residentes en los manicomios, propo-

35 Venancio, A.T.A.. (1990), Sobre a «nova psiquiatria» no Brasil: um estudo de caso do hospital-dia do Instituto de Psiquiatria. Dissertação de mestrado em antropologia social, Rio de Janeiro, Museu Nacional/UFRJ, p.115-116. 
niendo asimismo, como nuevo modelo, la reinserción social del enfermo mental y el fin de los manicomios. En este campo de la salud mental, las quejas fueron, ya a finales de la década de 1980, traducidas bajo la expresión de la ciudadanía del loco. La cuestión de la ciudadanía del loco ampliaba por fuerza el tema de la universalización y de la equidad propuesta por la reforma sanitaria, ya que buscaba cuestionar y reconocer la ciudadanía de ese sujeto «especial», frente a la comunidad basada en el contrato social. En este sentido, era necesario demarcar las limitaciones de este individuo frente a las exigencias racionales, la responsabilidad y la independencia socialmente impuestas, al mismo tiempo que se debería garantizar su ejercicio de la autonomía, la generación de renta propia, el derecho de libre circulación, etc.

Desde la segunda mitad de los años 80 , la reforma psiquiátrica dejó de ser predominantemente un movimiento de denuncia y crítica al modelo de asilos y pasó a producir resultados concretos dentro del espíritu que la animaba. Nuevos tipos de instituciones de servicio público - Hospitales de día, Núcleos y Centros de Atención Psicosocial (NAPS y CAPS), Clubs de Ocioempezaron a surgir en varios estados brasileños, todos como alternativas al ingreso y a la permanencia prolongada en hospitales psiquiátricos. Se observa a la vez una planificación de las políticas del sector en los años 90, cuando las directrices del Ministerio de la Salud, desde una coordinación que se identificaba con los ideales de la reforma, estimulaban esas experiencias asistenciales innovadoras por medio de una financiación distinta. Además, los pacientes y sus familiares, organizados en asociaciones, empezaron a participar en las conferencias municipales, regionales y nacionales de salud mental ${ }^{36}$.

Durante la década de 1990 varios estados brasileños — como Ceará, Pernambuco, Río Grande do Norte, Distrito Federal, Minas Gerais, Paraná y Río Grande do Sul - aprobaron legislaciones en pro de la sustitución progresiva de la asistencia y la internación hospitalaria por la atención en servicios extrahospitalarios, con vistas a un desarrollo permanente de la vida social del paciente, reconociendo así sus derechos civiles ${ }^{37}$. Esas legislaciones se inspiraban en un proyecto mayor de ley federal elaborado por los líderes del movimiento de reforma psiquiátrica y presentado a la Cámara de los Diputados del Congreso Nacional en 1989 por el Diputado Paulo Delgado del Partido de los Trabajadores que, sin embargo, encontró grandes dificultades para ser aprobado.

36 De acuerdo con TENóRIo, F.R. (2001), A Psicanálise e a Clínica da Reforma Psiquiátrica, Rio de Janeiro, Rios Ambiciosos; RuSSO e VenANCIO (2008).

37 BRASIL/ Ministério da Saúde /Secretaria Executiva. Legislação em saúde mental 1990-2002. 3ª ed. revista. Brasília: Ministério da Saúde, 2002. 
Se tramitó el proyecto de ley federal en el Congreso y en el Senado durante doce años, con la redacción de varios textos sustitutivos, frutos de una dura lucha política. En líneas generales, podemos decir que, de un lado, estaban los parlamentarios que defendían la idea de «una sociedad sin manicomios», junto con los que consideraban importante la reforma de la asistencia psiquiátrica, pero temían sus efectos; y de otro, estaban aquellos que se oponían a la transformación de la asistencia, abogando por la continuidad del modelo de tratamiento centrado en el ingreso psiquiátrico. En 2001, el proyecto de Ley federal fue votado y aprobado como Ley $n^{\circ} 10.216$. Su primer artículo destaca como objetivo el eliminar cualquier forma de discriminación de las personas con trastorno mental, en cuanto a la raza, el sexo, el color de la piel, la orientación sexual, la religión, la nacionalidad, la edad, los recursos económicos, etc. Inspirada en la ley italiana, determina los modos por los cuales han de tener lugar los ingresos: voluntario, involuntario o forzoso. La necesidad del consentimiento del paciente para ser ingresado, la idea de que el ingreso involuntario debe ser controlado por el Ministerio Público, o la determinación de que el carácter forzoso del ingreso debe considerar la salvaguarda del paciente, pretenden impedir los ingresos arbitrarios y la violación de sus derechos humanos y civiles. Quedaban entonces instauradas las bases sobre las cuales el tratamiento psiquiátrico - marcado por la idea de la ciudadanía del loco - debería ser defendido y ejercido: la garantía del derecho del paciente a negarse a recibir tratamiento, y el deber incontestable del Estado de ofrecerle y prestarle servicio.

\section{CONSIDERACIONES FINALES}

Este artículo ha buscado demostrar que la asistencia psiquiátrica en Brasil - en las dimensiones específicas de su planificación, organización y efectiva realización - fue fruto de las íntimas relaciones entre el campo psiquiátrico y el contexto político en el cual se dio. En este sentido, se ha buscado amplificar el argumento histórico de que la creación y la implantación de instituciones asistenciales psiquiátricas en Brasil, a ejemplo del proceso francés de constitución y desarrollo de la institución asilar, fue solamente, o principalmente, expresión de los amplios procesos de imposición disciplinaria propios de la modernidad, esos que produjeron un panorama decisivo de medicalización de la sociedad y de control social. Desde esta perspectiva, se ha pretendido construir un enfoque analítico más generalizador en relación con una concepción que remarca la medicina psiquiátrica como agente del poder dis- 
ciplinario. En este sentido, se han destacado los procesos históricos específicos del contexto brasileño, buscando mostrar los diferentes actores sociales e intereses en juego. Aunque sea incontestable el hecho de que, históricamente, la asistencia psiquiátrica en Brasil produjo un cuadro de exclusión social para una parcela significativa de la población, es necesario considerar que este proceso no solo no fue homogéneo temporal y espacialmente, sino que, además, fue simplemente uno de los desdoblamientos de otros procesos sociales y políticos más amplios que estaban teniendo lugar.

De esta forma, se ha señalado que la constitución del primer manicomio brasileño en 1841 formó parte de un proyecto político que tenía como objetivo consolidar los ideales monárquicos en el país. La creación de un manicomio, antes de ser un instrumento de control social fomentado por el proceso de medicalización de la sociedad, contribuía a la demostración de las alianzas entre el poder monárquico y el poder religioso, y al deseo de participar en el modelo de civilización europeo anclado en la misión de la caridad. Como vemos, en Brasil, la creación de la institución del manicomio no derivó de inmediato la gestión del problema de la locura a manos del cuerpo médico, aunque se estuviera de acuerdo con las recomendaciones de un grupo de médicos inspirados en la higiene, y tampoco llevó a la institucionalización del saber psiquiátrico en Brasil.

En esta misma línea interpretativa, parece demostrado que, en los albores del siglo XX, el proyecto de organización de la asistencia psiquiátrica formaba parte de una idea más amplia de reformulación de los espacios físicos y simbólicos de la ciudad de Río de Janeiro. La entonces capital de la República serviría como laboratorio para la implantación de un proyecto civilizador que pasaba a presentar el desafío — desde entonces continuamente presentede organizar una sociedad regida por los principios republicanos de igualdad y libertad. El orden político recientemente instaurado, marcado por esos nuevos valores, se veía, de esta manera, frente al desafío de sobreponerse a la tradición patriarcal y esclavista, que se mantenía viva en el imaginario y las prácticas sociales de la época. En este sentido, se ha destacado que la política asistencial resultó una aliada importante del proceso de institucionalización de la ciencia psiquiátrica en el ámbito brasileño, al mismo tiempo que el desarrollo y la consolidación de campos científicos eran considerados pilares centrales para el reconocimiento del grado de civismo de una sociedad.

La implantación de una política asistencial psiquiátrica de ámbito nacional en los años 40, por su parte, no provocó solo el incremento del número de plazas psiquiátricas en todo el país, considerado por la historiografía como una clara expresión de la extensión de métodos de control social, sino que 
trató también de hacer participar el área de la psiquiatría en el complejo desarrollo de una política de salud pública, buscando para tal objetivo el aumento de la cobertura asistencial a la población brasileña, diseminada por el amplio territorio nacional. Estaba en juego la implantación de una política pública que demostrara el liderazgo y el centralismo del gobierno federal en la constitución de un Estado nacional frente a las resistencias y las exigencias de los distintos estados brasileños.

Por último, se ha recalcado que solo en un contexto de distensión del régimen autoritario de la dictadura militar, pudo tener lugar el proceso de reforma psiquiátrica, aunque ya hubiese acontecido en otros países desde las décadas inmediatamente posteriores al final de la Segunda Guerra Mundial. En Brasil, ese proceso adquiere colores propios en cuanto a cómo ocurrió la apertura política y el uso de los canales e instrumentos democráticos a finales de la década de 1970. Se dio preferencia a las relaciones asociativas entre iguales; y la complejidad del juego político en el Congreso Nacional y en el Senado Federal no impidió las victorias de la reforma psiquiátrica en varios estados del país. Al contrario, de hecho parece que la reforma psiquiátrica tuvo repercusión y se consolidó por la vía de las experiencias locales, convirtiéndose más adelante en una propuesta nacional legislativa, defendida por el Gobierno Federal. En dicho contexto, la asistencia psiquiátrica se transformaría cada vez más en la negociación de las pretensiones de los diferentes actores sociales (médicos, otros profesionales del campo de la salud mental, pacientes y ex pacientes) que se resistían al modelo centrado en los ingresos, así como en el desarrollo de nuevas formas de reinserción social de los enfermos. Seguramente, esas propuestas han planteado nuevas cuestiones para la asistencia psiquiátrica brasileña, que sigue teniendo frente a ella el reto de responder - en un plazo de tiempo concreto - a los problemas propiamente estructurales de la modernidad, como el de crear, en el contexto de una comunidad basada en el contrato social, un lugar para la diferencia instituida como locura.

Recibido: 13 de enero de 2010

Aceptado: 1 de marzo de 2011 